Mon. Not. R. Astron. Soc. 000,1 17 7(2016) Printed 16 December $2016 \quad$ (MN LTEX style file v2.2)

\title{
Modeling the light curves of ultraluminous X-ray sources as precession
}

\author{
T. Dauser ${ }^{1 \star}$, M. Middleton ${ }^{2,3}$, J. Wilms ${ }^{1}$ \\ ${ }^{1}$ Dr. Karl Remeis-Observatory and Erlangen Centre for Astroparticle Physics, Sternwartstr. 7, 96049 Bamberg, Germany \\ ${ }^{2}$ Institute of Astronomy, Madingley Road, Cambridge CB3 OHA \\ ${ }^{3}$ School of Physics \& Astronomy, University of Southampton, Southampton, UK
}

16 December 2016

\begin{abstract}
We present a freely available XSPEC model for the modulations seen in the long-term light curves of multiple ultraluminous X-ray sources (ULXs). By incorporating the physics of multiple electron scatterings (ray traced with a Monte-Carlo routine), we go beyond analytical predictions and show that the geometrical beaming of radiation in the conical outflow can be more than a factor of 100 for opening angles smaller than $10^{\circ}$. We apply our new model to the longterm, well sampled $S$ wift light curve of the recently confirmed ULX pulsar NGC 5907 X-1 with an established period of 78 days. Our results suggest that geometrical beaming together with a slight precession of the conical wind can describe the light curve with a consistent set of parameters for the wind. The small opening angle of roughly $10-13^{\circ}$ implies a highly super-critical flow and boosting factors at the order of $\mathcal{B}=60-90$ that would yield a fairly low surface magnetic field strength of $2 \times 10^{10}$ Gauss.
\end{abstract}

Key words: accretion, accretion discs - black hole physics - X-rays: binaries - pulsars: individual (NGC 5907 ULX-1)

\section{INTRODUCTION}

Ultraluminous X-ray sources (ULXs) have traditionally been defined by both their apparent X-ray luminosities exceeding $1 \times$ $10^{39} \mathrm{erg} / \mathrm{s}$ and by their spectra showing a 'break' between 2 and $10 \mathrm{keV}$ (see Stobbart, Roberts \& Wilms 2006, Gladstone, Roberts \& Done 2009. Bachetti et al. 2013). More recently, their short (typically $<10 \mathrm{ksec}$ ) and long (months-years) timescale variability has been used as a complementary lever arm to diagnose the nature of the accretion flow with spectral-timing results suggesting that the majority of the bright $\left(>3 \times 10^{39} \mathrm{erg} / \mathrm{s}\right)$ ULXs can be successfully interpreted in the framework of super-critical accretion (see Shakura \& Sunyaev 1973, Poutanen et al. 2007) onto stellar remnants $\left(<100 M_{\odot}\right)$ (see Sutton, Roberts \& Middleton 2013, Middleton et al. 2015a, and references therein).

Numerical radiative magnetohydrodynamical (RMHD) studies of super-critical accretion (see Ohsuga 2007; Ohsuga \& Mineshige 2011; Jiang, Stone \& Davis 2013, Sa̧dowski et al. 2014) confirm the analytical prediction that the scale-height of the accretion flow is very large (typically $\sim 1$ ) when the mass transfer rate through the disc (typically $\gtrsim 10^{-6} M / 10 M_{\odot}$ ) results from unstable mass transfer occurring when the donor star evolves on a thermal timescale (King \& Begelman 1999, King \& Ritter 1999 King, Taam \& Begelman 2000, Podsiadlowski \& Rappaport 2000 Tauris, van den Heuvel \& Savonije 2000). The large scale-height

* E-mail: thomas.dauser@sternwarte.uni-erlangen.de disc and subsequently radiation pressure driven winds are expected to precess on long timescales (see Middleton et al., in prep). The predicted spectral-timing signature of such precession (as well as changes in accretion rate) was formalised in Middleton et al. (2015a). Such predictions are consistent with the observed spectraltiming evolution seen in canonical systems (e.g. HoIX ULX-1 (Luangtip, Roberts \& Done 2016), whilst additional evidence for precession comes from the correlation between the strength of atomic features - indicating an outflowing, mass loaded wind (Middleton et al. 2014, Pinto, Middleton \& Fabian 2016, Walton et al. 2016) — and the spectral hardness (Middleton et al. 2015b).

A corollary of such a large scale-height flow is that the emission from the inner regions is trapped and geometrically beamed out of the wind-cone (King 2009), which, depending on the opening angle should lead to orders of magnitude amplification in observed brightness. Such beaming must follow from the presence of an equatorial optically thick wind (Pinto, Middleton \& Fabian 2016) and is consistent with the general lack of eclipses in ULXs (Middleton \& King 2016, although see the recently discovered eclipsing ULXs by Urquhart \& Soria 2016).

Numerical simulations to-date have focused on how the radiation is generated in the flow (from dissipation due to magnetorotationally induced turbulence) and the impact of vertical as well as radial advection (Jiang, Stone \& Davis 2013) but not the impact of geometrical beaming as a function of the wind opening angle. In this paper we determine the observational impact of scattering off an optically thick wind cone and the shape such preces- 
sion should leave in the long timescale light curves of such sources (Sect. 2). In Sect. 4 we present an XSPEC local model built from our simulations that can be used to model the light curve variations. We then apply this model successfully to data of the ULX pulsar NGC 5907 ULX-1 in Sect. 5. before providing conclusions and a summary in Sect.6

\section{SIMULATIONS}

While radiation MHD simulations show the outflowing winds in ULXs to be fairly complex on small scales (e.g., Takeuchi, Ohsuga \& Mineshige 2013, Jiang, Stone \& Davis 2014; Sa̧dowski et al. 2014), they confirm the global geometry to be conical. In order to determine the impact of beaming for a given wind opening angle, we therefore employ a simple, but representative toy model. We assume that due to the extremely large accretion rate, the outflow lying above the radiation pressure supported accretion disc forms a cone and is fully ionised down to a Thomson depth greater than unity. Radiation emitted from the inner regions of the cone will be efficiently scattered off its surface. We do not consider the spectral changes (moderate Compton down-scattering) and so treat the geometrical beaming as achromatic. The scattering itself is modelled by the Klein-Nishina formula in a razor-thin layer of the conical surface, as will be described later in detail.

A sketch of the model geometry, including the definition of the parameters used to describe it, is depicted in Fig. 1 The geometry is very much in agreement with the model proposed by Middleton et al. (2015a Fig. 1), focusing only on the radiation emitted in the cone and the scattering off its surface. We parameterize the cone by the angle, $\theta$, between the rotational axis and the surface of the outflow. The opening angle of the cone is therefore $2 \theta$. The height of the cone is described by the parameter $h_{\text {cone }}$ which according to standard theory is related to the physical properties of the accretion flow by $h_{\text {cone }} \sim \dot{m} r_{\text {in }}$, where $\dot{m}$ is the mass inflow rate through the disc, and $r_{\text {in }}$ is the inner disc radius (approximately the ISCO). Note that this relation is only valid for the inflow (where the scaleheight is radiation pressure supported and approximately unity) and does not necessarily hold for the direction and height of the outflow.

We assume the emission to originate isotropically from a disclike region located $h_{\text {emit }}$ above the central object (as shown in Figure 1), whilst $h_{\text {cone }} \gg h_{\text {emit }}$. This is an approximation to emission from the region where the outflow is optically thin (see Poutanen et al. 2007). As we will see, the resulting light curve is insensitive to the exact shape of the emission region. The emission itself is isotropic in all directions, i.e., also towards the black hole. The simulation and calculations for the model are performed using Monte Carlo simulations in three dimensions. The observer's inclination to the system is defined to be the angle between the rotational axis of the black hole and the line-of-sight.

We locate the emitter at a height of $10 r_{\mathrm{g}}$ to be of order the radial location of the inner photosphere $R_{\mathrm{ph} \text {,in }}$ (see Poutanen et al. 2007), where the outflow is starting to become optically thick and form the surface of the cone in our model. This has the benefit of being large enough such that general relativistic effects are secondary to the scattering processes taking place in the cone and can be safely ignored. The photons are therefore assumed to travel on straight, Euclidean, trajectories between their interactions with the cone.

The physics of the surface of the accretion disc, especially in such an inflated state is currently not well constrained. As discussed in the introduction, due to the large quantity of photons trapped in-

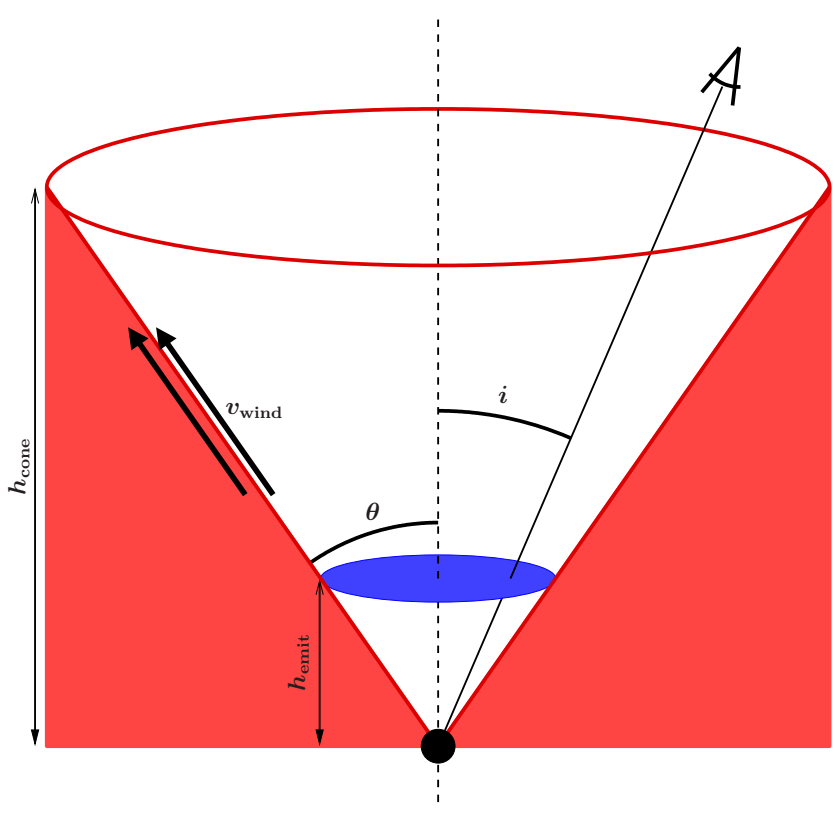

Figure 1. Geometrical model of the super-critical accretion disc (drawn in red), labelling the important parameters of the model, the cone's half opening angle, $\theta$, the height of the cone, $h_{\text {cone }}$, and the observer's inclination angle, $i$. The emitting region is shown as the blue region, which is located at a height $h_{\text {emit }}$ above the black hole. The velocity of the wind at the surface of the cone is given by $v_{\text {wind }}$.

side the cone it is expected that the surface is highly ionised (values expected up to $\xi>10^{6}$, see, e.g., Middleton et al. 2015a). We therefore assume that the scattering on the surface of the cone is purely due to electron scattering, described using the Klein-Nishina differential cross section. While each encounter of a photon with the surface of the cone would actually lead to multiple scatterings inside the surface layer, in the current approach this is simplified to a single scattering event on a razor thin surface. This simplification can be made, as we are only interested in the direction of the photon and therefore the Klein-Nishina angle characteristic of the scattering will still be conserved. Note that choosing an isotropic scattering profile instead, does not largely influence the simulation results. A full treatment of the scattering including the energies is out of scope for this paper and will be subject of future investigations.

The simulation also includes special relativistic boosting of the photons, in order to model an outflowing surface of the cone (see Poutanen et al.2007). It is taken into account by transforming the direction of a given photon from the rest frame of the cone, where the scattering is simulated, to the global, non-moving frame of the system. Rough observational constraints (lower limits) on the wind speed lie between $v \approx 0.2-0.4 c$ (see, e.g., Middleton et al. 2014, Pinto, Middleton \& Fabian 2016).

We note that emission from the outflow itself (i.e. scattered emission from the underlying thick disc) is ignored. This is a reasonable first order assumption as the inner regions are those from which emission is most likely to be beamed.

\section{RESULTS}

The simulation set-up described above directly predicts the viewing angle-dependent flux from a specific system. The results for different parameter combinations are shown in Fig. 2 As mentioned in 
the previous section, velocities of the outflow are expected to be in the range of $v \approx 0.2-0.4 c$ and we consider a height of the cone of $1000 r_{\mathrm{g}}$ as plausible. Note that even much larger values have been suggested for SS433 (Fabrika \& Medvedev 2011). Currently there do not exist any constraints on the opening angle of such a cone.

The number of scatterings a single photon exhibits until escaping the cone strongly depends on the opening angle of the cone. In cones with small opening angles of $10^{\circ}$ photons will usually scatter around $\sim 50$ times. This number quickly drops to only around $\sim 15$ scatterings for $20^{\circ}$, and 1 scattering on average for $45^{\circ}$. The exact number of scatterings depends on the height of the cone and also the velocity of the outflow.

We determine the effect of the boosting in the wind-cone by normalising the outgoing radiation relative to the flux of an isotropic source, defining the flux boost as

$$
\mathcal{B}=L_{\text {iso }}(i) / L
$$

where $L$ is the actual luminosity of the source, and $L_{\text {iso }}(i)$ the luminosity measured at a certain inclination $i$ to the system assuming an isotropically emitting source. The latter value is generally quoted as the observed luminosity for ULXs due to the lack of geometrical information. Therefore $\mathcal{B}$ actually describes the amount by which the luminosity would be overestimated ${ }^{1}$

Figure 2 a shows the flux boost for a stationary cone and different opening angles. It is immediately evident that for $i \leqslant \theta$, i.e., when we directly look into the cone, the observed flux is constant, but boosted strongly depending on the opening angle. For values of $\theta$ as small as $5^{\circ}$, an effective flux boost of up to a factor $\approx 100$ is possible simply by collimation. If the observer is not looking directly into the cone, the observed flux drops quickly below the value expected for isotropic emission. For larger observer inclination angles (i.e. $i \geqslant \theta$ ) the emissivity profile does not depend on the opening angle.

Besides the system inclination, the emissivity profile also depends on the geometrical and physical properties of the cone. The two parameters mainly influencing the profile are the velocity of the outflow (Fig. 2p) and the vertical extent of the cone (Fig. 22 ). Generally the dependence of the boost on both parameters is not very strong, although a large parameter space is explored in the plots. Notably, a change in the height of the cone will be strongly degenerate with the normalisation of the curve and therefore introduce an uncertainty in the determination of the boosting factor. However, the inclination angle at which the steep drop in emissivity occurs is unaffected. The flux boost when looking directly into the cone can be large and of the order of a factor $\approx 2$ for velocities up to $v=0.7 \mathrm{c}$. Differences between the outflowing velocities at larger inclination angles are very small and hard to detect with current detector sensitivities. We also note that even a slightly different shape of the cone or variability might also produce differences of similarly small magnitudes.

From the previous figures we can conclude that there is a flat part of the emissivity profile when we look directly into the cone, where, due to scattering on the outflowing walls of the cone, the intensity is geometrically beamed. Assuming that we observe a source when looking into the cone, the apparent maximal flux boost $\mathcal{B}_{\max }$ for a certain configuration can be calculated. Figure 3 shows the flux boost for a stationary and outflowing wind at a height of $1000 r_{\mathrm{g}}$. While for very narrow cones a factor of a few hundred in flux boost is possible, even for an opening angle of $30^{\circ}$ (i.e.,

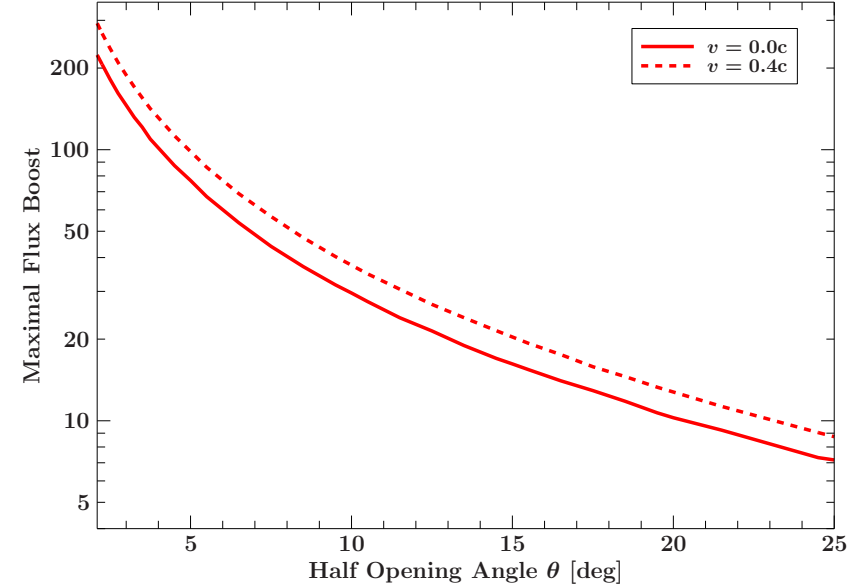

Figure 3. This plot visualises the maximal flux boost possible for a certain values of $\theta$ (half opening angle). Different combinations for a stationary (solid) and fast moving surface (dashed) of the cone are shown.

$\theta=15^{\circ}$ ) a source would be observed at a factor of $\approx 10$ boosted compared to its isotropic luminosity.

\section{PRECESSING DISCS IN ULX}

Observations of ULXs have revealed roughly periodic flux variations on the order of months in sources such as M82 X-1, (Kaaret, Simet \& Lang 2006), M82 X-2 (Kong et al. 2016) and NGC 5907 ULX-1 (Walton et al. 2016). The latter source has very recently been identified as a pulsar accreting at a highly superEddington rate (Israel et al. 2016a, Fuerst et al. 2016b). Using the above model of geometrical beaming in the cone of the outflow, such a behaviour can be readily explained, either by changes in the line-of-sight to the observer due to precession of the binary orbit, or (as suggested in the case of M82 X-1 Pasham \& Strohmayer (2013)), more likely by a precession of the accretion disc. The existence of precessing discs has long been known (e.g. in Her X-1, see Petterson 1977) and also investigated from a theoretical point of view (see Pringle 1996, Maloney \& Begelman 1997, Maloney, Begelman \& Nowak 1998, Fragile et al. 2007). Recent studies beyond the basic theory suggest that also self-gravity of the disc influences the precession in the case of supermassive black holes (Tremaine \& Davis 2014). In addition, the low-frequency quasiperiodic oscillations (QPOs) found in binaries have been recently connected to Lense-Thirring precession of the accretion flow (see Ingram, Done \& Fragile 2009. Ingram \& Done 2011).

We now study whether we can reproduce similar light curves using a precessing inflow/outflow, where the inclination with respect to the accretion disc's angular momentum vector (or the system orbit) is varying. We will designate this variation by $\Delta i$, i.e., effectively the system is viewed from angles in the range from $i-\Delta i$ to $i+\Delta i$.

Using the approach outlined above, we constructed an XSPEC local model (Arnaud 1996). The model is publicly available for download ${ }^{2}$ and can be readily used in any common X-ray data analysis software. The emissivity profiles are precalculated in a table on a fine grid, which allows for a fast evaluation. The height of the accretion disc is fixed to $h_{\text {cone }}=1000 r_{\mathrm{g}}$, as for reasonable heights 


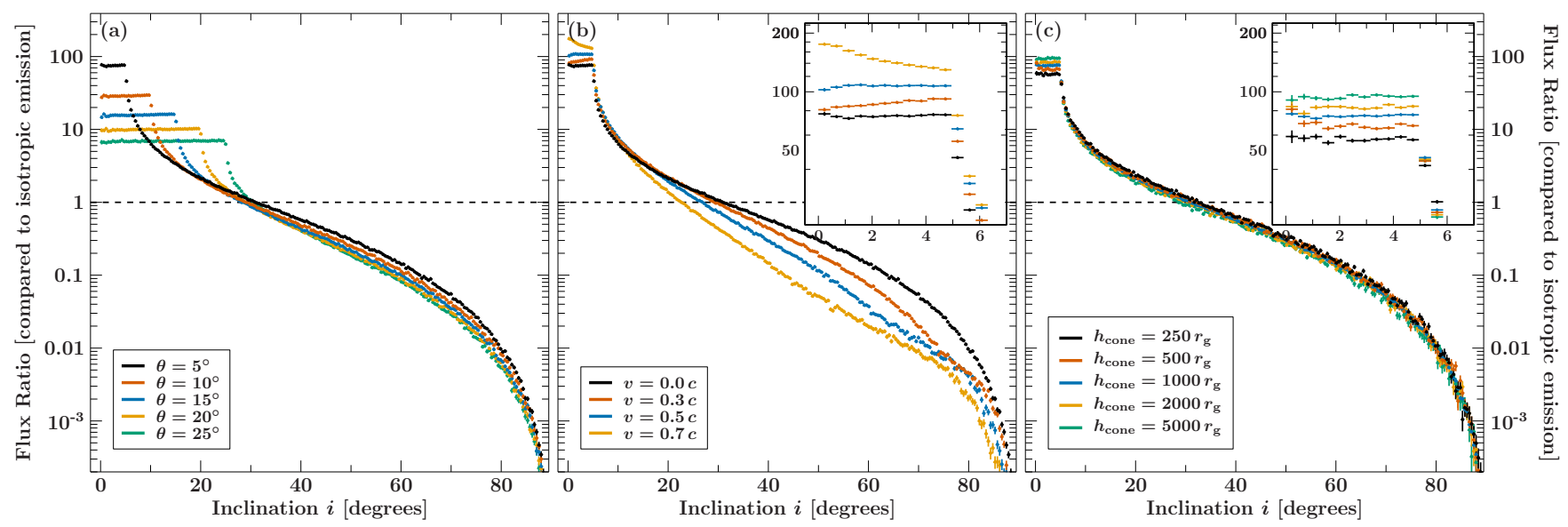

Figure 2. Flux emerging from the outflow compared to an isotropically emitting source, shown for different inclination angles (a) Variation of the of the flux amplification as a function of the half-opening angle, $\theta$. The height of the cone is chosen at an intermediate value of $h_{\text {cone }}=1000 r_{\mathrm{g}}$. (b) Variation of the flux for different velocities of the cone, assuming fixed height of the cone of $h_{\text {cone }}=1000 r_{\mathrm{g}}$ and $\theta=5^{\circ}$. The inset shows a zoom into the low inclination angle region. (c) Emissivity profile for a non-moving surface $(v=0)$ for increasing height $h_{\text {cone }}$ of the cone, and again $\theta=5^{\circ}$.

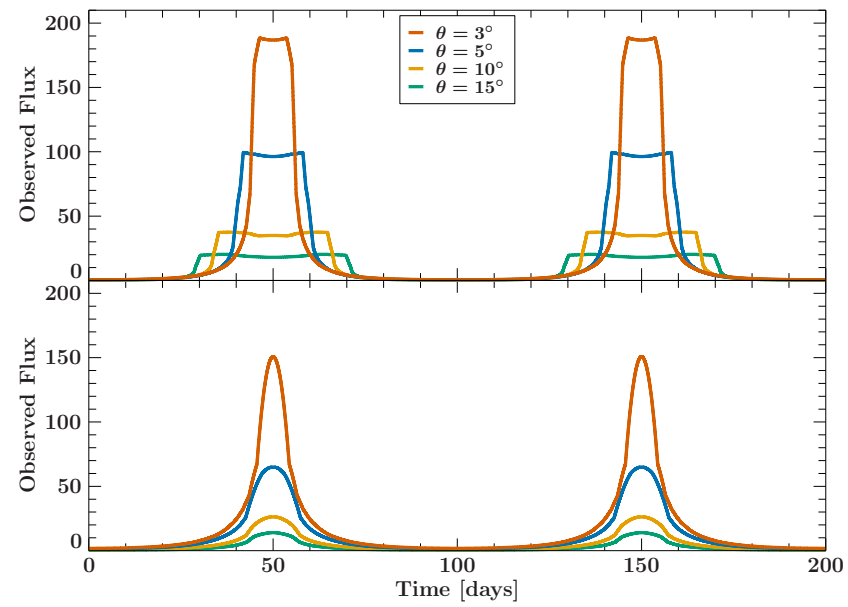

Figure 4. Simulated light curves with the ulxlc model, for a velocity of $0.4 c$, fixing the height of the cone to $h_{\text {cone }}=1000 r_{\mathrm{g}}$. The orbit is arbitrarily assumed to be 100 days and we show the light curve for two precession periods for clarity. Different colours are for different half opening angles, $\theta$, as indicated in the plot. Upper panel: Light curve for a larger viewing angle $\left(i=20^{\circ}\right)$ and larger precession angle amplitude $\Delta i=18^{\circ}$. Lower panel: Varying inclination angle according to $i=\theta+10^{\circ}$ with a fixed precession angle $\Delta i=10^{\circ}$.

the parameter is largely degenerate with changes in the flux normalisation and does not have a large effect on the overall emissivity profile (see Fig. 2).

An example of the model in action for certain parameter combinations is shown in Fig 4 As expected, the duration of the peaks increases with opening angle whilst the strength decreases. For extreme precession angles, a double peaked profile is in principle possible (as the observers line-of-sight passes fully over the face of the cone).

Note that the light curve predicted in this model assumes a constant mass accretion rate, $\dot{m}$. If $\dot{m}$ is varying, an additional flux variation is expected (Poutanen et al. 2007). In order to model light curves, we will assume that changes in $\dot{m}$ are small enough to be ignored (or that variations at the spherisation radius are long com- pared to the precession) such that the intrinsic luminosity is constant over a precession period.

\section{APPLICATION TO DATA}

From the predicted light curves (see Sect. 4), it is clear that small opening angles of around $10^{\circ}$ are necessary in order to explain the boosting from a moderate luminosity of about the Eddington luminosity, $L_{\mathrm{Edd}}$, to an observed luminosity of larger than $100 L_{\mathrm{Edd}}$.

In the following we apply our model to NGC 5907 ULX-1 (recently confirmed as a pulsar Israel et al. 2016a). As revealed by high-cadence Swift monitoring observations spanning several years, the source shows a remarkable pattern of variability, with repeated brightenings on timescales of $\sim 78$ days (Walton et al. 2016) and observed luminosities of over $100 L_{\mathrm{Edd}}$ (for a canonical neutron star mass). We obtain the data from the UK Swift science data centre (UKSSDC ${ }^{3}$ ) for the XRT in photon counting mode and rebin the resulting light curves to 4 days/bin. We convert the light curve into pha format using the FTOOL FLX2XSP which also creates a flat response file.

First we obtain the light curve in soft $(0.3-1 \mathrm{keV})$ and hard (1$10 \mathrm{keV}$ ) energy bands in order to verify that the hard X-rays coming from the innermost accretion flow indeed produces the long-term variations. Figure 5 a show both light curves where it can clearly be seen that the quasi-periodic peaks are only found in the hard (red) light curve, which is also largely dominating the total observed flux. We can therefore safely apply our model to the full-band light curve (as the soft emission only provides a constant offset).

The light curve with the best fit model is shown in Fig. 6 In order to obtain reasonable results we had to allow a shift in phase between the different precession periods. Namely the 5 th and the 6th peak are shifted by a $\Delta$ phase $\sim 0.2$ (i.e. $\sim 14$ days) with respect to the preceding peaks (this was also visible in the light curve fitting of Walton et al. 2016). Afterwards, the remaining three peaks coincide directly with the first peaks (see Fig. 67). Such a behaviour is not unreasonable given that the precession period should be dependent on $\dot{m}$ (Middleton et al. in prep).

3 http://www.swift.ac.uk 


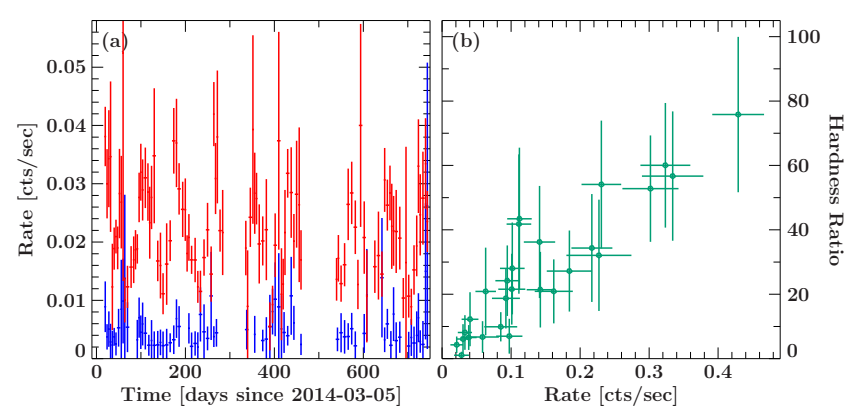

Figure 5. Left: long-term Swift light curves of the hard band $(1-10 \mathrm{keV}$, red) and soft (0.3-1 keV, blue) band of NGC 5907 ULX-1. The binning is 4 days. It can be seen that the hard light curve, which shows the quasi-periodic peaks, dominates the total observed flux of the source. Right: hardness ratio of the $S$ wift count rate in the $1-10 \mathrm{keV}$ band divided by the $0.3-1 \mathrm{keV}$ band plotted against the total count rate. To increase the statistics, a binning of 25 days was chosen (close to $1 / 3$ of the precession period of this pulsar). A clear hardening for larger count rates is evident.

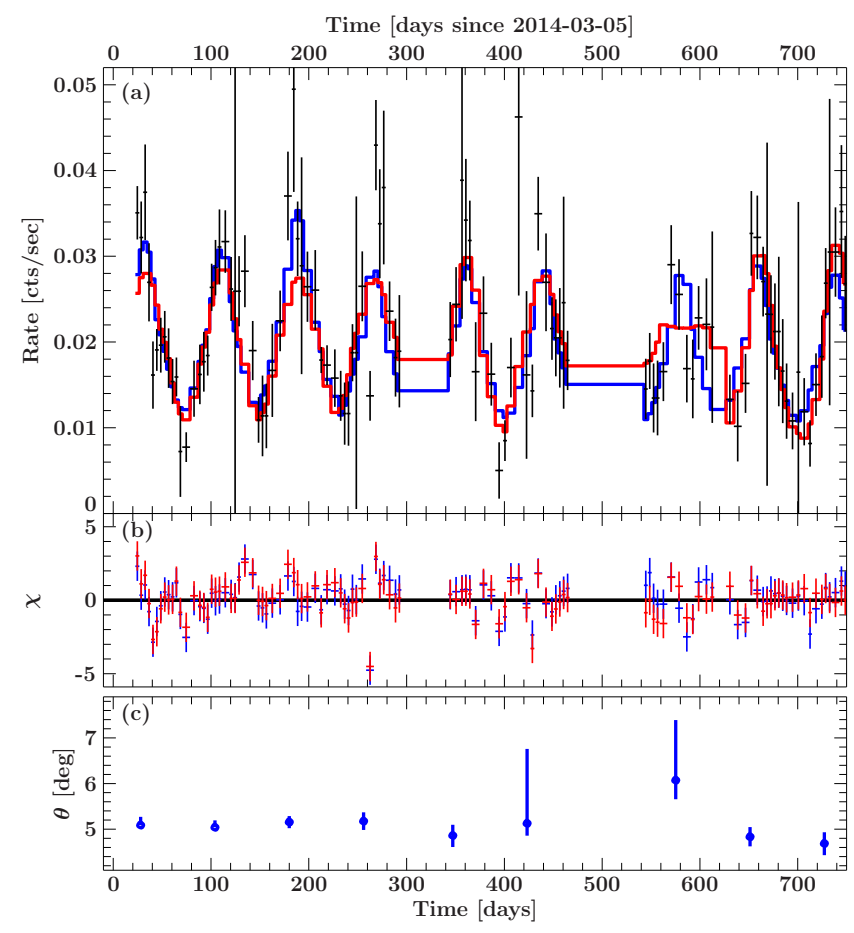

Figure 6. (a) The 0.3-10 keV Swift XRT light curve of NGC 5907 ULX-1, binned to 4 days. The model with a fixed opening angle is shown in red (model 1), while the model with variable opening angle is shown in blue (model 2). (b) Residuals of the model fits depicted in the same colours. (c) The half opening angles $\theta$ of the best fit model with a fixed intrinsic luminosity (blue curve in (a), model 2). In most cases the half opening angle is around 5 degrees.

As discussed above, we assume that there are no long-term changes in $\dot{m}$ and we initially assume that the $i, \theta$, and the precessing angle $\Delta i$ are constant, but that the intrinsic source luminosity can vary between individual precession cycles. Additionally we use a fixed period of 78.1 days for the precession period (Walton et al. 2016.

Fits to the light curve show that the flux variation between different precession cycles are all within $\pm 20 \%$ of each other and so we assume that the variation of $\dot{m}$ does not influence the geometry, but only the normalisation of the peaks (i.e. the intrinsic luminosity of the object changes). Therefore in our initial fit (model 1) we assume the geometry to be stable and obtain a global half opening angle $\theta$. As shown in Fig. 6 this model is able to describe the data with a goodness-of-fit of $\chi^{2} /$ d.o.f. $=156.2 / 94=1.66$. We find an inclination of $i=6.40_{-0.02}^{+0.22}$ degrees and a precession angle of $\Delta i=7.30_{-0.15}^{+0.13}$ degrees. The half opening angle is determined to be $\theta=6.70_{-0.11}^{+2.31}$ degrees. The velocity of the outflow could not be well constrained. The boosting factor determined from this fit, assuming a representative outflow velocity of $v=0.2 c$ is $\mathcal{B} \approx 58$.

In a second, complementary fit (model 2), we assume that the intrinsic luminosity is constant. Therefore the change in shape and normalisation of the peaks will solely be described by a change in the opening angle, i.e., the parameter $\theta$. Using these assumptions we can find a slightly better fit $\chi^{2} /$ d.o.f. $=149.0 / 94=1.58$ ). In this case we find a smaller inclination of $i=5.02_{-0.07}^{+0.04}$ with $\Delta i=0.76_{-0.18}^{+0.15}$.

Figure 6r shows how the opening angle varies over the different peaks for the model with a fixed (intrinsic) normalisation (model 2). Generally, opening angles are found mainly around $10^{\circ}$ (i.e., half opening angles around $\theta=5^{\circ}$ ) with the opening angle remaining quite stable between the different precessing periods. In the case of a slightly smaller opening angle, we derive a large boosting factor of $\mathcal{B} \approx 87$ for this model, again assuming a representative outflow velocity of $v=0.2 \mathrm{c}$.

From Fig. 6a it can be seen that both models are able to describe the data well and predict a similar shape for the peaks. The relatively poor reduced $\chi^{2}$ values are mainly driven by a few outlier data points, which clearly do not follow the general trend of each peak. Therefore we conclude that the simple geometrical light curve model is able to reproduce the overall shape of the light curve well.

These results suggest that NGC 5907 ULX-1 is viewed almost face-on and explains the hard X-ray spectrum (see Middleton et al. 2015a). Due to the extremely large accretion rate, it might be that the emission comes only from a very small cone with an opening angle of roughly 10 degrees. With our estimated boosting factor $(\mathcal{B}=60-90)$, the observed luminosity of $L_{\text {iso }} \approx 8.5 \times 10^{40} \mathrm{erg} / \mathrm{s}$ (for a distance of $17.1 \mathrm{Mpc}$, see Fuerst et al. 2016b for more details) is reduced significantly to an intrinsically emitted luminosity of $L=1.0-1.5 \times 10^{39} \mathrm{erg} / \mathrm{s}$. Super-critical discs have luminosities which follow $L_{E d d}(1+\ln (\dot{m})$ ) (Shakura \& Sunyaev 1973) implying mass accretion rates into the disc of $\lesssim 100-1000$ times the Eddington rate $\left(\sim 1 \times 10^{-6}-1 \times 10^{-5} M_{\odot} / y r\right.$ assuming a radiative efficiency of 0.1 ) consistent with thermal timescale mass transfer (e.g. King \& Begelman 1999, King \& Ritter 1999).

\section{SUMMARY AND CONCLUSIONS}

In this paper we have presented a simple model for scattering in conical outflows, which are believed to exist in sources with a super-critical accretion rate. Importantly, our model includes a physical scattering process and so is more accurate than an analytical approach. For radiation emitted close to the black hole, our model predicts strong geometrical beaming of the radiation when viewing into the cone. This beaming can lead to an effective flux boost of up to a factor of 100 and, in cases where the conical outflow is moving at observed speeds $(\geqslant 0.2-0.4 \mathrm{c}$ Middleton et al. 2014 Pinto, Middleton \& Fabian 2016) this value can be far higher. In order to reach such large boosting factors, opening angles of 10 degrees and smaller are necessary. We note, however, that for more 
moderate opening angles of up to 30 degrees boosting factors of up to 10 can still be achieved, hinting that also the larger class of ULX sources observed around $L_{\text {iso }}=1 \times 10^{39} \mathrm{erg} / \mathrm{s}$ could at least partly be explained by the proposed geometrical beaming model (although the majority are likely to be associated with the tail of the normal X-ray binary population: Swartz et al. 2011, Soria et al. 2012, Middleton et al. 2013).

Observational proof that stellar compact objects are able to produce such luminosities has recently tightened through the discovery that the ULXs M82 X-2 (Bachetti et al. 2013), NGC 7793 P13 (Fuerst et al. 2016a; Israel et al. 2016b) and NGC 5907 ULX-1 (Israel et al.|2016a) are pulsars accreting at more than $100 L_{\mathrm{Edd}}$. We conclude that the model we presented is easily able to explain the observed flux boost for these sources. Contrary to the statement of (Fuerst et al. 2016b) that the observed smooth pulse profiles in such objects (see also Fuerst et al.|2016a, Bachetti et al. 2014) is at odds with such a narrow cone, we suggest that the different lengths of the photon trajectory due to the multiple scatterings must smoothen the pulse profile of the intrinsic source.

The XSPEC model ulxlc constructed from the simulations was successfully fitted to the long-term light curve of the ULX pulsar NGC 5907 ULX-1. Within our model the system is found to be viewed almost face-on with only a small precession angle needed to explain the large quasi-periodic flux changes. We employed two complimentary model assumptions and show that the observed difference in the peaks of the light curve can be solely explained by intrinsic source variability or a change in the opening angle. We note that clearly those must be linked to some degree as the opening angle is driven by changes in mass accretion rate. The opening angle determined from the fits range from $10^{\circ}-13^{\circ}$, resulting in a boosting factor of roughly $60-90$ and mass accretion rates $\lesssim 100$ $1000 \times$ Eddington.

The obtained boosting factor and almost face-on geometry now strongly support the interpretation of NGC 5907 ULX-1 with a low surface magnetic field. Following Fuerst et al. (2016b Eq. 14) and King \& Lasota (2016), we can estimate the magnetospheric radius $\approx 120 R_{\mathrm{g}}$, by using their spectral fitting results and $\cos (i) \approx 1$. Employing the obtained intrinsic luminosities, we derive a quite low magnetic field strength of $B \approx 2 \times 10^{10} \mathrm{G}$ in this case. The above results use a pulsed fraction of $20 \%$ (see Israel et al. 2016a), which is comparably low for a high-mass X-ray binary (see Bildsten et al.|1997), but supporting our model where the hard X-ray radiation is produced at the inner regions close to the neutron star (see also Kluźniak \& Lasota 2015).

Our simple modelling of the conical surface requires a smooth and constant outflow, which is not expected in real RMHD flows which are subject to instabilities (Jiang, Stone \& Davis 2014. Sagdowski et al. 2014) although the resulting stratification is expected to be small scale (Takeuchi, Ohsuga \& Mineshige 2013) and can therefore be ignored to first order. However, a more dedicated analysis of light curves, the connection to the mass accretion rate, and the expected spectral changes due to the scattering in the cone are necessary in order to further strengthen our findings.

Acknowledgements. TD acknowledges funding by the Deutsches Zentrum für Luft- und Raumfahrt contract 50 QR 1402. MJM acknowledges funding from STFC via an Ernest Rutherford advanced grant. We thank Matthias Kühnel for valuable discussions and John E. Davis for the development of the SLXFIG module used to prepare the figures in this letter. This research has made use of ISIS functions provided by ECAP/Remeis observatory and MIT (http://www.sternwarte.uni-erlangen.de/isis/).

\section{REFERENCES}

Arnaud K. A., 1996, in Astronomical Data Analysis Software and Systems V, G. H. Jacoby \& J. Barnes, ed., Astron. Soc. Pacific Conf. Ser. 101, pp. 17-20

Bachetti M. et al., 2014, Nat, 514, 202

Bachetti M. et al., 2013, ApJ, 778, 163

Bildsten L. et al., 1997, ApJS, 113, 367

Fabrika S., Medvedev A., 2011, in Jets at All Scales, Romero G. E., Sunyaev R. A., Belloni T., eds., pp. 280-284

Fragile P. C., Blaes O. M., Anninos P., Salmonson J. D., 2007, ApJ, 668, 417

Fuerst F. et al., 2016a, submitted to ApJ, arXiv:1609.07129

Fuerst F., Walton D. J., Stern D., Bachetti M., Barret D., Brightman M., Harrison F. A., Rana V., 2016b, submitted to ApJ, arXiv: 1610.00258

Gladstone J. C., Roberts T. P., Done C., 2009, MNRAS, 397, 1836

Ingram A., Done C., 2011, MNRAS, 415, 2323

Ingram A., Done C., Fragile P. C., 2009, MNRAS, 397, L101

Israel G. L. et al., 2016a, ArXiv e-prints, submitted, arXiv:1609.07375

Israel G. L. et al., 2016b, submitted to MNRAS, arXiv:1609.06538

Jiang Y.-F., Stone J. M., Davis S. W., 2013, ApJ, 778, 65

Jiang Y.-F., Stone J. M., Davis S. W., 2014, ApJ, 796, 106

Kaaret P., Simet M. G., Lang C. C., 2006, Science, 311, 491

King A., Lasota J.-P., 2016, MNRAS, 458, L10

King A. R., 2009, MNRAS, 393, L41

King A. R., Begelman M. C., 1999, Astrophys. J., Lett., 519, L169

King A. R., Ritter H., 1999, MNRAS, 309, 253

King A. R., Taam R. E., Begelman M. C., 2000, Astrophys. J., Lett., 530, L25

Kluźniak W., Lasota J.-P., 2015, MNRAS, 448, L43

Kong A. K. H., Hu C.-P., Lin L. C.-C., Li K. L., Jin R., Liu C. Y., Yen D. C.-C., 2016, MNRAS, 461, 4395

Luangtip W., Roberts T. P., Done C., 2016, MNRAS, 460, 4417

Maloney P. R., Begelman M. C., 1997, Astrophys. J., Lett., 491, L43

Maloney P. R., Begelman M. C., Nowak M. A., 1998, ApJ, 504, 77

Middleton M. J., Heil L., Pintore F., Walton D. J., Roberts T. P., 2015a, MNRAS, 447, 3243

Middleton M. J., King A., 2016, MNRAS, 462, L71

Middleton M. J. et al., 2013, Nat, 493, 187

Middleton M. J., Walton D. J., Fabian A., Roberts T. P., Heil L., Pinto C., Anderson G., Sutton A., 2015b, MNRAS, 454, 3134

Middleton M. J., Walton D. J., Roberts T. P., Heil L., 2014, MNRAS, 438, L51

Ohsuga K., 2007, ApJ, 659, 205

Ohsuga K., Mineshige S., 2011, ApJ, 736, 2

Pasham D. R., Strohmayer T. E., 2013, Astrophys. J., Lett., 774, L16

Petterson J. A., 1977, ApJ, 218, 783

Pinto C., Middleton M. J., Fabian A. C., 2016, Nat, 533, 64

Podsiadlowski P., Rappaport S., 2000, ApJ, 529, 946

Poutanen J., Lipunova G., Fabrika S., Butkevich A. G., Abolmasov P., 2007, MNRAS, 377, 1187

Pringle J. E., 1996, MNRAS, 281, 357

Sa̧dowski A., Narayan R., McKinney J. C., Tchekhovskoy A., 2014, MNRAS, 439, 503

Shakura N. I., Sunyaev R. A., 1973, A\&A, 24, 337

Soria R., Kuntz K. D., Winkler P. F., Blair W. P., Long K. S., 
Plucinsky P. P., Whitmore B. C., 2012, ApJ, 750, 152

Stobbart A.-M., Roberts T. P., Wilms J., 2006, MNRAS, 368, 397

Sutton A. D., Roberts T. P., Middleton M. J., 2013, MNRAS, 435, 1758

Swartz D. A., Soria R., Tennant A. F., Yukita M., 2011, ApJ, 741, 49

Takeuchi S., Ohsuga K., Mineshige S., 2013, PASJ, 65, 88

Tauris T. M., van den Heuvel E. P. J., Savonije G. J., 2000, Astrophys. J., Lett., 530, L93

Tremaine S., Davis S. W., 2014, MNRAS, 441, 1408

Urquhart R., Soria R., 2016, ApJ, 831, 56

Walton D. J. et al., 2016, Astrophys. J., Lett., 827, L13 